Volume 10, No.3, May - June 2021

International Journal of Advanced Trends in Computer Science and Engineering Available Online at http://www.warse.org/IJATCSE/static/pdf/file/ijatcse1421032021.pdf

https://doi.org/10.30534/ijatcse/2021/1431032021

\title{
Python program to generate spherical harmonic
}

\author{
Kousar Shaheen $^{1}$, Roohi Zafar ${ }^{1}$, Saba Javaid ${ }^{1}$ \\ ${ }^{1}$ Department of Physics, NED University of Engineering and Technology, Pakistan \\ kousarshaheen416@gmail.com \\ roohizj@neduet.edu.pk \\ hars4@yahoo.com ,
}

\begin{abstract}
A generalized Python program has been developed to show pictorial form of wave function of hydrogen and hydrogen like atoms. This program will be helpful to teach solution of Schrodinger equation for hydrogen and hydrogen like atoms to undergraduate students. The python program not only shows pictorial form of the wave function but it also gives analytical form of the wave function. The wavefunctions and the orbital from 1s $(1,0,0)$ to $9 f(9,3,0)$ are presented in this article.
\end{abstract}

Key words: Probability Distribution Function, Associated Laguerre Polynomials, Associated Legendre Polynomials, Hyper geometric function.

\section{INTRODUCTION}

Hydrogen atom is of fundamental important, as it is the only atom for which Schrödinger's equation can be solved exactly and the wave function of this atom provides the basis for many approximate methods to solve many electron atoms. It has been found that students, which are studied in introduction of Quantum Physics, misinterpret polar graphs and hydrogen eigen state probability distribution plots. In 2002, Budde et.al investigated that according to their research, experience, gaining knowledge of quantum atomic model increases many problems. Detailed survey of various college students' preconceptions problems may be found [1,2]. In 2002, Müller et.al found that many college students believe that in atom just like the Bohr's model, electrons orbiting around the nucleus. Although a number of them recognize that it's not correct, this idea appears to be very resistant [3]. There are new approaches to solve these troubles and lead college students to better understanding [2-4]. In 1962, Fowles et.al generated a general equation of Cartesian coordinate with modification of Schrodinger's equation by separable variable method and making assumption that a wave function depends upon four variables $\mathrm{x}, \mathrm{y}, \mathrm{z}, \mathrm{r}$ [5]. The Mathematica software program offers a variety of tools for understanding, manipulate and visualize atomic and Molecular systems, in which, orthogonal polynomials and Clebsch- Gordan Coefficients, polar plots, spherical plots, density plots, Contour plots and animation are included [6-7]. In 2013, Chung et.al described that how Winplot software programs can be used to understand and visualize micorbitals and wave functions of a hydrogen like atom [8]. In this work, we transformed probability distribution function from Polar coordinate system to cartesian coordinate system by modifying spherical harmonics and radial distribution function. [9-11]

\section{THEORY}

Schrodinger equation for hydrogen and hydrogen like atom is a fundamental equation in quantum mechanics to solve wave functions for these atoms, which describes the state of the system. The wave equation for one electron system in the field of a nucleus of charge $\mathrm{Ze}$ is given by equation

$$
H \psi=\left(-\frac{\hbar^{2}}{2 m} \nabla^{2}-\frac{Z e^{2}}{r}\right) \psi=E \psi
$$

Where $\mathrm{E}$ is the total energy of the system, $\psi$ is the total wavefunction and $\mathrm{Z}$ is the atomic number. Since coulomb potential energy and the boundary conditions are spherically symmetric,solutions to Schrödinger's equation may be solved by using separation of variable method and the wavefunction can be written as the product of a radial and an angular part

$$
\psi(r, \theta, \varphi)=\frac{1}{r} P(n l, r) Y_{l m}(\theta, \varphi)
$$

2

Where $Y_{l m}(\theta, \varphi)$ a spherical harmonics with 1 is orbital quantum number, an integer with values $l \geq 0$. 


$$
Y_{l m}(\theta, \varphi)=\sqrt{\left(\frac{2 l+1}{4 \pi}\right) \frac{(l-|m|) !}{(l+|m|) !}} P_{l}^{m}(\cos \theta) e^{i m \varphi} \quad(m \geq 0)
$$

Where $P_{l}^{m}$ is a Legendre polynomial and can be calculated by using equation 4

\begin{tabular}{|c|l|}
\hline$P_{l}^{m}(\cos \theta)=\frac{(l+|m|) !}{(l-|m|) !} \frac{\left(1-\cos ^{2} \theta\right)^{\frac{|m|}{2}}}{2^{|m|}|m| !}{ }_{2} F_{1}\left(|m|-l,|m|+l+1 ;|m|+1 ; \frac{1-\cos \theta}{2}\right)$ & 4 \\
\hline${ }_{2} F_{1}\left(|m|-l,|m|+l+1 ;|m|+1 ; \frac{1-\cos \theta}{2}\right)=\sum_{k=0}^{\mathrm{n}+1+|\mathrm{m}|} \frac{(|m|-l)_{(k)}(|m|+l+1)_{(k)}}{(|m|+1)_{(k)}} \frac{\left(\frac{1-\cos \theta}{2}\right)^{k}}{k !}$ & 5 \\
\hline
\end{tabular}

The radial part of equation $2, P(n l, r)$ is a solution of the differential equation

$$
\left(\frac{d^{2}}{d r^{2}}+\frac{2 Z}{r}-\frac{l(l+1)}{r^{2}}-\frac{1}{n^{2}}\right) P(n l, r)=0
$$

With boundary conditions $P(n l, 0)=0$ and $P(n l, 0)=\infty$. For these boundary conditions only integer values of $\mathrm{n}$, principle quantum number, are allowed. Furthermore, the value of orbital quantum number 1 must be less than principle quantum number $n$. Number of nodes in the solution other than origin is $n-1-1$. We can calculate the radial function for the state with quantum number $n, l$ by using equation 7 .

$$
P(n l, r)=\sqrt{\left(\frac{2}{n a}\right)^{3} \frac{(n-l-1) !}{2 n[(n+t) !]^{3}}} e^{-\frac{r}{n a}}\left(\frac{2 r}{n a}\right)^{l}\left[L_{n-l-1}^{2 l+1}\left(\frac{2 r}{n a}\right)\right]
$$

7

In which $L_{n-l-1}^{2 l+1}$ is an associated Laguerre polynomial of degree n-1-1.

$$
L_{n-l-1}^{2 l+1}\left(\frac{2 r}{n a_{0}}\right)=(n+l) ! \sum_{j=0}^{n-l-1}(-1)^{j} \frac{(n+l) !}{(n-l-1-j) !(2 l+1+j) ! j !}\left(\frac{2 r}{n a}\right)^{j}
$$

Now by using equation 3-8, total wave function can be written as [11-13].

$$
\begin{aligned}
& \psi_{n l m}=\sqrt{\left(\frac{2}{n a}\right)^{3} \frac{(n-l-1) !}{2 n[(n+t) !]^{3}}} e^{-\frac{r}{n a}}\left(\frac{2 r}{n a}\right)^{l}\left[\sum_{j=0}^{n-l-1}(-1)^{j} \frac{(n+l) !}{(n-l-1-j) !(2 l+1+j) ! j !}\left(\frac{2 r}{n a}\right)^{j}\right] \\
& (n+l) ! \sqrt{\left(\frac{2 l+1}{4 \pi}\right) \frac{(l-|m|) !}{(l+|m|) !}} e^{i m \varphi} \frac{(l+|m|) !}{(l-|m|) !} \frac{\left(1-\cos ^{2} \theta\right)^{|m| / 2}}{2^{|m|}|m| !} \\
& \times \sum_{k=0}^{\mathrm{n}+1+|\mathrm{m}|} \frac{(|m|-l)_{(k)}(|m|+l+1)_{(k)}}{(|m|+1)_{(k)}} \frac{\left(\frac{1-\cos \theta}{2}\right)^{k}}{k !}
\end{aligned}
$$

\section{METHODOLOGY}

In this work by using transformation equation

\begin{tabular}{|c|l|}
\hline$r=\sqrt{x^{2}+y^{2}+z^{2}}$ & 10 \\
\hline$\theta=\arccos \frac{z}{\sqrt{x^{2}+y^{2}+z^{2}}}$ & 11 \\
\hline$\varphi=\arctan \frac{y}{x}$ & 12 \\
\hline
\end{tabular}


We can determine the explicit forms of associated Laguerre Polynomials, radial probability distribution and Hypergeometric function for Associated Legendre Polynomials into Cartesian coordinates and with the help of them; we can transform total wave function into Cartesian coordinate [14]

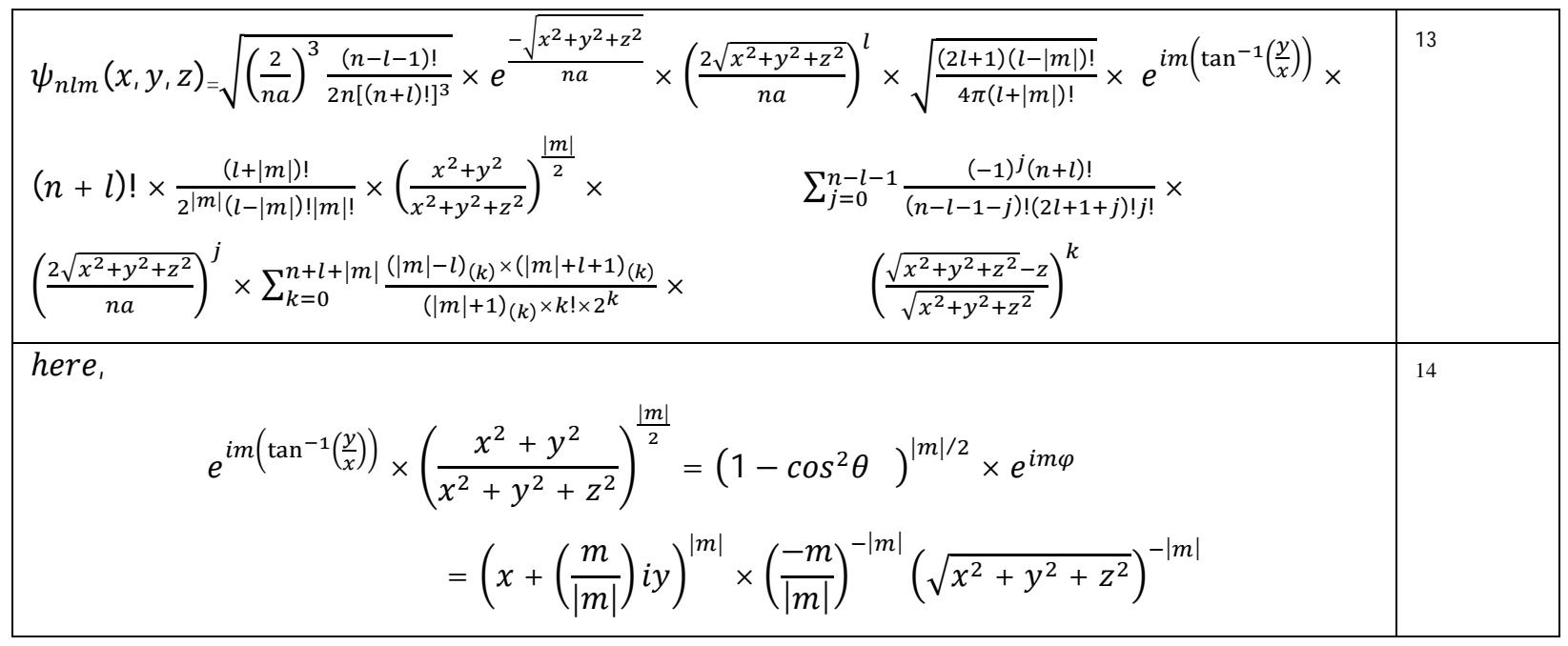

\begin{tabular}{|l|l|l|}
$\Psi_{n l m}(x, y, z)=\sqrt{\left(\frac{2}{n a}\right)^{3} \frac{(n-l-1) !}{2 n[(n+l) !]^{3}}} \times e^{\frac{-\sqrt{x^{2}+y^{2}+z^{2}}}{n a}} \times\left(\frac{2 \sqrt{x^{2}+y^{2}+z^{2}}}{n a}\right)^{l} \times \sqrt{\frac{(2 l+1)(l-|m|) !}{4 \pi(l+|m|) !} \times}$ & 15 \\
$\frac{(l+|m|) !}{2^{|m|}(l-|m|) !|m| !} \times\left(x+\left(\frac{m}{|m|}\right) i y\right)^{|m|} \times$ & $\sum_{j=0}^{n-l-1} \frac{(-1)^{j}(n+l) !}{(n-l-1-j) !(2 l+1+j) ! j !} \times\left(\frac{2 \sqrt{x^{2}+y^{2}+z^{2}}}{n a}\right)^{j} \times$ \\
$\sum_{k=0}^{n+l+|m|} \frac{(|m|-l)_{(k)} \times(|m|+l+1)_{(k)}}{(|m|+1)_{(k)} \times k ! \times 2^{k}} \times$ & $\left(\frac{\sqrt{x^{2}+y^{2}+z^{2}}-z}{\sqrt{x^{2}+y^{2}+z^{2}}}\right)^{k} \times\left(\frac{-m}{|m|}\right)^{-|m|}\left(\sqrt{x^{2}+y^{2}+z^{2}}\right)^{-|m|}$
\end{tabular}

The notations in lower subscript are pochhammer symbols e.g. $(\mathrm{m}-\mathrm{n})_{(\mathrm{k})}$ etc.

\section{RESULT AND DISCUSSION}

The general form of total wave function in Cartesian coordinate, equation (15), helps us to understand visualize the behavior of orbitals and probability distribution functions. In this work forty Wave functions from $(1,0,0)$ to $(9,3,0)$ are generated and are given in table 1. Some wave functions in Cartesian coordinates have also been plotted by using different tools of python plotting in python program which we have code see appendix[15].

Table 1: Wave functions from $(1,0,0)$ to $(9,3,0)$ in Cartesian coordinate

\begin{tabular}{|c|c|}
\hline$\Psi_{(1,0,0)}=0.707106781186548 \sqrt{2} \sqrt{\frac{1}{a^{3}}} e^{-\frac{\sqrt{x^{2}+y^{2}+z^{2}}}{a}} \div \sqrt{\pi}$ & 16 \\
\hline$\Psi_{(2,0,0)}=\sqrt{2}\left(0.25 a-0.125 \sqrt{x^{2}+y^{2}+z^{2}}\right) \sqrt{\frac{1}{a^{3}}} e^{-\frac{\sqrt{x^{2}+y^{2}+z^{2}}}{2 a}} \div(\sqrt{\pi} a)$ & 17 \\
\hline$\Psi_{(2,1,0)}=0.125 \sqrt{2} z \sqrt{\frac{1}{a^{3}}} e^{-\frac{\sqrt{x^{2}+y^{2}+z^{2}}}{2 a}} \div(\sqrt{\pi} a)$ & 18 \\
\hline $\begin{array}{l}\Psi \\
\sqrt{2}\left(0.136 a^{2}-0.09072 a \sqrt{x^{2}+y^{2}+z^{2}}+0.01008 x^{2}+0.01008 y^{2}+0.01008 z^{2}\right) \sqrt{\frac{1}{a^{3}}} e^{-\frac{\sqrt{x^{2}+y^{2}+z^{2}}}{3 a}} \div \\
\left(\sqrt{\pi} a^{2}\right)\end{array}$ & 19 \\
\hline$\Psi_{(3,1,0)}=0.61237 \sqrt{2} z\left(0.12096 a-0.02016 \sqrt{x^{2}+y^{2}+z^{2}}\right) \sqrt{\frac{1}{a^{3}}} e^{-\frac{\sqrt{x^{2}+y^{2}+z^{2}}}{3 a}} \div\left(\sqrt{\pi} a^{2}\right)$ & 20 \\
\hline
\end{tabular}




\begin{tabular}{|c|c|}
\hline$\Psi_{(3,2,0)}=\sqrt{2}\left(-0.00356389 x^{2}-0.00356389 y^{2}+0.00712778 z^{2}\right) \sqrt{\frac{1}{a^{3}}} e^{-\frac{\sqrt{x^{2}+y^{2}+z^{2}}}{3 a}} \div\left(\sqrt{\pi} a^{2}\right)$ & 21 \\
\hline $\begin{array}{l}\Psi_{(4,0,0)}=\sqrt{2}\left(0.0884 a^{3}-0.06629 a^{2} \sqrt{x^{2}+y^{2}+z^{2}}+0.0110485 a\left(x^{2}+y^{2}+z^{2}\right)-0.00046\left(x^{2}+\right.\right. \\
\left.\left.y^{2}+z^{2}\right)^{\frac{3}{2}}\right) \sqrt{\frac{1}{a^{3}}} e^{-\frac{\sqrt{x^{2}+y^{2}+z^{2}}}{4 a}} \div\left(\sqrt{\pi} a^{3}\right)\end{array}$ & 22 \\
\hline $\begin{array}{l}\Psi_{(4,1,0)}=0.61237 \sqrt{2} z\left(0.080687 a^{2}-0.02017 a \sqrt{x^{2}+y^{2}+z^{2}}+0.0010086 x^{2}+0.0010086 y^{2}+\right. \\
\left.0.0010086 z^{2}\right) \sqrt{\frac{1}{a^{3}}} e^{-\frac{\sqrt{x^{2}+y^{2}+z^{2}}}{4 a}} \div\left(\sqrt{\pi} a^{3}\right)\end{array}$ & 23 \\
\hline $\begin{array}{l}\Psi_{(4,2,0)}=0.790569 \sqrt{2}\left(0.0069877 a-0.00058 \sqrt{x^{2}+y^{2}+z^{2}}\right)\left(3.0 z\left(x^{2}+y^{2}+z^{2}\right)+1.5(z-\right. \\
\left.\left.\sqrt{x^{2}+y^{2}+z^{2}}\right)^{2} \sqrt{x^{2}+y^{2}+z^{2}}-2.0\left(x^{2}+y^{2}+z^{2}\right)^{\frac{3}{2}}\right) \sqrt{\frac{1}{a^{3}}} e^{-\frac{\sqrt{x^{2}+y^{2}+z^{2}}}{4 a}} \div\left(\sqrt{\pi} a^{3} \sqrt{x^{2}+y^{2}+z^{2}}\right)\end{array}$ & 24 \\
\hline $\begin{array}{l}\Psi_{(4,3,0)}=\sqrt{2}\left(0.001235 z\left(x^{2}+y^{2}+z^{2}\right)^{\frac{5}{2}}+0.0005147\left(z-\sqrt{x^{2}+y^{2}+z^{2}}\right)^{3}\left(x^{2}+y^{2}+z^{2}\right)^{\frac{3}{2}}+\right. \\
\left.0.0015441\left(z-\sqrt{x^{2}+y^{2}+z^{2}}\right)^{2}\left(x^{2}+y^{2}+z^{2}\right)^{2}-0.0010294\left(x^{2}+y^{2}+z^{2}\right)^{3}\right) \sqrt{\frac{1}{a^{3}}} e^{-\frac{\sqrt{x^{2}+y^{2}+z^{2}}}{4 a}} \div \\
\left(\sqrt{\pi} a^{3}\left(x^{2}+y^{2}+z^{2}\right)^{\frac{3}{2}}\right)\end{array}$ & 25 \\
\hline $\begin{array}{c}\Psi_{(5,0,0)}=\sqrt{2}\left(0.063246 a^{4}-0.050596 a^{3} \sqrt{x^{2}+y^{2}+z^{2}}+0.0101193 a^{2}\left(x^{2}+y^{2}+z^{2}\right)-\right. \\
\left.0.0006746 a\left(x^{2}+y^{2}+z^{2}\right)^{\frac{3}{2}}+1.349 \times 10^{-5}\left(x^{2}+y^{2}+z^{2}\right)^{2}\right) \sqrt{\frac{1}{a^{3}}} e^{-\frac{\sqrt{x^{2}+y^{2}+z^{2}}}{5 a}} \div\left(\sqrt{\pi} a^{4}\right)\end{array}$ & 26 \\
\hline $\begin{array}{l}\Psi_{(5,1,0)}=0.61237 \sqrt{2} z\left(0.0584 a^{3}-0.01753 a^{2} \sqrt{x^{2}+y^{2}+z^{2}}+0.0014 a\left(x^{2}+y^{2}+z^{2}\right)\right. \\
\left.3.1159 \times 10^{-5}\left(x^{2}+y^{2}+z^{2}\right)^{\frac{3}{2}}\right) \sqrt{\frac{1}{a^{3}}} e^{-\frac{\sqrt{x^{2}+y^{2}+z^{2}}}{5 a}} \div\left(\sqrt{\pi} a^{4}\right)\end{array}$ & 27 \\
\hline $\begin{array}{l}\Psi_{(5,2,0)}=0.79057 \sqrt{2}\left(3.0 z\left(x^{2}+y^{2}+z^{2}\right)+1.5\left(z-\sqrt{x^{2}+y^{2}+z^{2}}\right)^{2} \sqrt{x^{2}+y^{2}+z^{2}}-2.0\left(x^{2}+\right.\right. \\
\left.\left.y^{2}+z^{2}\right)^{\frac{3}{2}}\right)\left(0.005355 a^{2}-0.00071395 a \sqrt{x^{2}+y^{2}+z^{2}}+2.03986 \cdot 10^{-5} x^{2}+2.03986 \cdot 10^{-5} y^{2}+\right. \\
\left.2.03986 \cdot 10^{-5} z^{2}\right) \sqrt{\frac{1}{a^{3}}} e^{-\frac{\sqrt{x^{2}+y^{2}+z^{2}}}{5 a}} \div\left(\sqrt{\pi} a^{4} \sqrt{x^{2}+y^{2}+z^{2}}\right)\end{array}$ & 28 \\
\hline $\begin{array}{l}\Psi_{(5,3,0)}=0.9354 \sqrt{2}\left(0.000204 a-1.01993 \cdot 10^{-5} \sqrt{x^{2}+y^{2}+z^{2}}\right)\left(6.0 z\left(x^{2}+y^{2}+z^{2}\right)^{\frac{5}{2}}+\right. \\
2.5\left(z-\sqrt{x^{2}+y^{2}+z^{2}}\right)^{3}\left(x^{2}+y^{2}+z^{2}\right)^{\frac{3}{2}}+7.5\left(z-\sqrt{x^{2}+y^{2}+z^{2}}\right)^{2}\left(x^{2}+y^{2}+z^{2}\right)^{2}- \\
\left.5.0\left(x^{2}+y^{2}+z^{2}\right)^{3}\right) \sqrt{\frac{1}{a^{3}}} e^{-\frac{\sqrt{x^{2}+y^{2}+z^{2}}}{5 a}} \div\left(\sqrt{\pi} a^{4}\left(x^{2}+y^{2}+z^{2}\right)^{\frac{3}{2}}\right)\end{array}$ & 29 \\
\hline $\begin{array}{l}\Psi_{(5,4,0)}=\sqrt{2}\left(3.60599 \cdot 10^{-5} z\left(x^{2}+y^{2}+z^{2}\right)^{\frac{9}{2}}+1.5776 \cdot 10^{-5}\left(z-\sqrt{x^{2}+y^{2}+z^{2}}\right)^{4}\left(x^{2}+y^{2}+\right.\right. \\
\left.z^{2}\right)^{3}+6.310485 \cdot 10^{-5}\left(z-\sqrt{x^{2}+y^{2}+z^{2}}\right)^{3}\left(x^{2}+y^{2}+z^{2}\right)^{\frac{7}{2}}+8.11348 \times \\
\left.10^{-5}\left(z-\sqrt{x^{2}+y^{2}+z^{2}}\right)^{2}\left(x^{2}+y^{2}+z^{2}\right)^{4}-3.24539 \cdot 10^{-5}\left(x^{2}+y^{2}+z^{2}\right)^{5}\right) \sqrt{\frac{1}{a^{3}}} e^{-\frac{\sqrt{x^{2}+y^{2}+z^{2}}}{5 a} \div} \\
\left(\sqrt{\pi} a^{4}\left(x^{2}+y^{2}+z^{2}\right)^{3}\right)\end{array}$ & 30 \\
\hline $\begin{array}{l}\Psi_{(6,0,0)} \\
\sqrt{2}\left(0.04811 a^{5}-0.040094 a^{4} \sqrt{x^{2}+y^{2}+z^{2}}+0.00891 a^{3}\left(x^{2}+y^{2}+z^{2}\right)-0.0007425 a^{2}\left(x^{2}+\right.\right. \\
\left.\left.y^{2}+z^{2}\right)^{\frac{3}{2}}+2.474924 \cdot 10^{-5} a\left(x^{2}+y^{2}+z^{2}\right)^{2}-2.74992 \cdot 10^{-7}\left(x^{2}+y^{2}+z^{2}\right)^{\frac{5}{2}}\right) \sqrt{\frac{1}{a^{3}}} e^{-\frac{\sqrt{x^{2}+y^{2}+z^{2}}}{6 a} \div} \\
\left(\sqrt{\pi} a^{5}\right)\end{array}$ & 31 \\
\hline $\begin{array}{l}\Psi_{(6,1,0)}=0.61237 \sqrt{2} z\left(0.04473 a^{4}-0.01491 a^{3} \sqrt{x^{2}+y^{2}+z^{2}}+0.001491 a^{2}\left(x^{2}+y^{2}+z^{2}\right)-\right. \\
\left.5.522 \cdot 10^{-5} a\left(x^{2}+y^{2}+z^{2}\right)^{\frac{3}{2}}+6.574 \cdot 10^{-7}\left(x^{2}+y^{2}+z^{2}\right)^{2}\right) \sqrt{\frac{1}{a^{3}}} e^{-\frac{\sqrt{x^{2}+y^{2}+z^{2}}}{6 a}} \div \quad\left(\sqrt{\pi} a^{5}\right)\end{array}$ & 32 \\
\hline
\end{tabular}




\begin{tabular}{|c|c|}
\hline $\begin{array}{l}\Psi_{(6,2,0)}=0.7905 \sqrt{2}\left(3.0 z\left(x^{2}+y^{2}+z^{2}\right)+1.5\left(z-\sqrt{x^{2}+y^{2}+z^{2}}\right)^{2} \sqrt{x^{2}+y^{2}+z^{2}}-2.0\left(x^{2}+\right.\right. \\
\left.\left.y^{2}+z^{2}\right)^{\frac{3}{2}}\right)\left(0.00421685 a^{3}-0.00070281 a^{2} \sqrt{x^{2}+y^{2}+z^{2}}+3.3467 \cdot 10^{-5} a\left(x^{2}+y^{2}+z^{2}\right)-\right. \\
\left.4.6482 \cdot 10^{-7}\left(x^{2}+y^{2}+z^{2}\right)^{\frac{3}{2}}\right) \sqrt{\frac{1}{a^{3}}} e^{-\frac{\sqrt{x^{2}+y^{2}+z^{2}}}{6 a}} \div\left(\sqrt{\pi} a^{5} \sqrt{x^{2}+y^{2}+z^{2}}\right)\end{array}$ & 33 \\
\hline $\begin{array}{l}\Psi_{(6,3,0)}=0.9354 \sqrt{2}\left(6.0 z\left(x^{2}+y^{2}+z^{2}\right)^{\frac{5}{2}}+2.5\left(z-\sqrt{x^{2}+y^{2}+z^{2}}\right)^{3}\left(x^{2}+y^{2}+z^{2}\right)^{\frac{3}{2}}+7.5(z-\right. \\
\left.\left.\sqrt{x^{2}+y^{2}+z^{2}}\right)^{2}\left(x^{2}+y^{2}+z^{2}\right)^{2}-5.0\left(x^{2}+y^{2}+z^{2}\right)^{3}\right)\left(0.0001739 a^{2}-1.4492 \cdot\right. \\
10^{-5} a \sqrt{x^{2}+y^{2}+z^{2}}+2.68364 \\
\left.\times 10^{-7} x^{2}+2.68364 \cdot 10^{-7} y^{2}+2.68364 \cdot 10^{-7} z^{2}\right) \sqrt{\frac{1}{a^{3}}} e^{-\frac{\sqrt{x^{2}+y^{2}+z^{2}}}{6 a}} \div\left(\sqrt{\pi} a^{5}\left(x^{2}+y^{2}+z^{2}\right)^{\frac{3}{2}}\right)\end{array}$ & 34 \\
\hline $\begin{array}{l}\Psi_{(6,4,0)} \\
\sqrt{2}\left(3.6005 \cdot 10^{-6} a-1.20016 \cdot 10^{-7} \sqrt{x^{2}+y^{2}+z^{2}}\right)\left(10.0 z\left(x^{2}+y^{2}+z^{2}\right)^{\frac{9}{2}}+4.375 \quad(z-\right. \\
\left.\sqrt{x^{2}+y^{2}+z^{2}}\right)^{4}\left(x^{2}+y^{2}+z^{2}\right)^{3}+17.5\left(z-\sqrt{x^{2}+y^{2}+z^{2}}\right)^{3}\left(x^{2}+y^{2}+z^{2}\right)^{\frac{7}{2}}+22.5 \quad(z- \\
\left.\left.\sqrt{x^{2}+y^{2}+z^{2}}\right)^{2}\left(x^{2} 36+y^{2}+z^{2}\right)^{4}-9.0\left(x^{2}+y^{2}+z^{2}\right)^{5}\right) \sqrt{\frac{1}{a^{3}}} e^{-\frac{\sqrt{x^{2}+y^{2}+z^{2}}}{6 a}} \div\left(\sqrt{\pi} a^{5}\left(x^{2}+y^{2}+\right.\right. \\
\left.\left.z^{2}\right)^{3}\right)\end{array}$ & 35 \\
\hline $\begin{array}{l}\Psi_{(6,5,0)}=\sqrt{2}\left(6.36482 \cdot 10^{-7} z\left(x^{2}+y^{2}+z^{2}\right)^{7}+3.34153 \cdot 10^{-7}\left(z-\sqrt{x^{2}+y^{2}+z^{2}}\right)^{5}\left(x^{2}+y^{2}+\right.\right. \\
\left.z^{2}\right)^{5}+1.670765 \cdot 10^{-6}\left(z-\sqrt{x^{2}+y^{2}+z^{2}}\right)^{4}\left(x^{2}+y^{2}+z^{2}\right)^{\frac{11}{2}}+2.97025 \times 10^{-6}(z- \\
\left.\sqrt{x^{2}+y^{2}+z^{2}}\right)^{3}\left(x^{2}+y^{2}+z^{2}\right)^{6}+2.2277 \cdot 10^{-6}\left(z-\sqrt{x^{2}+y^{2}+z^{2}}\right)^{2}\left(x^{2}+y^{2}+z^{2}\right)^{\frac{13}{2}}- \\
\left.5.940496 \cdot 10^{-7}\left(x^{2}+y^{2}+z^{2}\right)^{\frac{15}{2}}\right) \sqrt{\frac{1}{a^{3}}} e^{-\frac{\sqrt{x^{2}+y^{2}+z^{2}}}{6 a}} \div\left(\sqrt{\pi} a^{5}\left(x^{2}+y^{2}+z^{2}\right)^{5}\right)\end{array}$ & 36 \\
\hline $\begin{array}{l}\Psi_{(7,0,0)} \\
\sqrt{2}\left(0.0382 a^{6}-0.032726 a^{5} \sqrt{x^{2}+y^{2}+z^{2}}+0.007792 a^{4}\left(x^{2}+y^{2}+z^{2}\right)-0.0007421 a^{3}\left(x^{2}+\right.\right. \\
\left.y^{2}+z^{2}\right)^{\frac{3}{2}}+3.180356 \cdot 10^{-5} a^{2}\left(x^{2}+y^{2}+z^{2}\right)^{2}-\quad 6.05782 \times 10^{-7} a\left(x^{2}+y^{2}+z^{2}\right)^{\frac{5}{2}}+4.120967 . \\
\left.10^{-9}\left(x^{2}+y^{2}+z^{2}\right)^{3}\right) \sqrt{\frac{1}{a^{3}}} e^{-\frac{\sqrt{x^{2}+y^{2}+z^{2}}}{7 a}} \div\left(\sqrt{\pi} a^{6}\right)\end{array}$ & 37 \\
\hline $\begin{array}{l}\Psi_{(7,1,0)}=0.61237 \sqrt{2} z\left(0.035627 a^{5}-0.0127241 a^{4} \sqrt{x^{2}+y^{2}+z^{2}}+0.001454 a^{3}\left(x^{2}+y^{2}+z^{2}\right)-\right. \\
\quad 6.9247 \cdot 10^{-5} a^{2}\left(x^{2}+y^{2}+z^{2}\right)^{\frac{3}{2}}+1.413197 \cdot 10^{-6} a\left(x^{2}+y^{2}+z^{2}\right)^{2}-1.00943 \cdot 10^{-8}\left(x^{2}+y^{2}+\right. \\
\left.\left.z^{2}\right)^{\frac{5}{2}}\right) \sqrt{\frac{1}{a^{3}}} e^{-\frac{\sqrt{x^{2}+y^{2}+z^{2}}}{7 a}} \div\left(\sqrt{\pi} a^{6}\right)\end{array}$ & 38 \\
\hline $\begin{array}{l}\Psi_{(7,2,0)}=0.790569 \sqrt{2}\left(3.0 z\left(x^{2}+y^{2}+z^{2}\right)+1.5\left(z-\sqrt{x^{2}+y^{2}+z^{2}}\right)^{2} \sqrt{x^{2}+y^{2}+z^{2}}-2.0\left(x^{2}+\right.\right. \\
\left.\left.y^{2}+z^{2}\right)^{\frac{3}{2}}\right)\left(0.0034 a^{4}-0.00065 a^{3} \sqrt{x^{2}+y^{2}+z^{2}}+3.98161 \cdot 10^{-5} a^{2}\left(x^{2}+y^{2}+z^{2}\right)-\right. \\
\left.9.4800157 \cdot 10^{-7} a\left(x^{2}+y^{2}+z^{2}\right)^{\frac{3}{2}}+7.52382 \cdot 10^{-9}\left(x^{2}+y^{2}+z^{2}\right)^{2}\right) \sqrt{\frac{1}{a^{3}}} e^{-\frac{\sqrt{x^{2}+y^{2}+z^{2}}}{7 a}} \div \\
\left(\sqrt{\pi} a^{6} \sqrt{x^{2}+y^{2}+z^{2}}\right)\end{array}$ & 39 \\
\hline $\begin{array}{l}\Psi_{(7,2,0)} 0.790569 \sqrt{2}\left(3.0 z\left(x^{2}+y^{2}+z^{2}\right)+1.5\left(z-\sqrt{x^{2}+y^{2}+z^{2}}\right)^{2} \sqrt{x^{2}+y^{2}+z^{2}}-2.0\left(x^{2}+y^{2}+\right.\right. \\
\left.\left.z^{2}\right)^{\frac{3}{2}}\right)\left(0.0034 a^{4}-0.00065 a^{3} \sqrt{x^{2}+y^{2}+z^{2}}+3.98161 \cdot 10^{-5} a^{2}\left(x^{2}+y^{2}+z^{2}\right)-9.4800157\right. \\
\left.10^{-7} a\left(x^{2}+y^{2}+z^{2}\right)^{\frac{3}{2}}+7.52382 \cdot 10^{-9}\left(x^{2}+y^{2}+z^{2}\right)^{2}\right) \sqrt{\frac{1}{a^{3}}} e^{-\frac{\sqrt{x^{2}+y^{2}+z^{2}}}{7 a}} \div\left(\sqrt{\pi} a^{6} \sqrt{x^{2}+y^{2}+z^{2}}\right)\end{array}$ & 40 \\
\hline $\begin{array}{l}\Psi_{(7,3,0)}=\quad 0.9354 \sqrt{2}\left(0.00014689 a^{3}-1.573868 \cdot 10^{-5} a^{2} \sqrt{x^{2}+y^{2}+z^{2}}+4.99641 \cdot 10^{-7} a\left(x^{2}+\right.\right. \\
\left.\left.y^{2}+z^{2}\right)-4.7584828 \cdot 10^{-9}\left(x^{2}+y^{2}+z^{2}\right)^{\frac{3}{2}}\right)\left(6.0 z\left(x^{2}+y^{2}+z^{2}\right)^{\frac{5}{2}}+\right. \\
2.5\left(z-\sqrt{x^{2}+y^{2}+z^{2}}\right)^{3}\left(x^{2}+y^{2}+z^{2}\right)^{\frac{3}{2}}+7.5\left(z-\sqrt{x^{2}+y^{2}+z^{2}}\right)^{2}\left(x^{2}+y^{2}+z^{2}\right)^{2}-\end{array}$ & 41 \\
\hline
\end{tabular}




\begin{tabular}{|c|c|}
\hline $\left.5.0\left(x^{2}+y^{2}+z^{2}\right)^{3}\right) \sqrt{\frac{1}{a^{3}}} e^{-\frac{\sqrt{x^{2}+y^{2}+z^{2}}}{7 a}} \div\left(\sqrt{\pi} a^{6}\left(x^{2}+y^{2}+z^{2}\right)^{\frac{3}{2}}\right)$ & \\
\hline $\begin{array}{l}\Psi_{(7,4,0)}=1.06066 \sqrt{2}\left(3.34859 \cdot 10^{-6} a^{2}-1.913478 \cdot 10^{-7} a \sqrt{x^{2}+y^{2}+z^{2}}+2.48504 \cdot 10^{-9} x^{2}+\right. \\
\left.2.48504 \cdot 10^{-9} y^{2}+2.48504 \cdot 10^{-9} z^{2}\right)\left(10.0 z\left(x^{2}+y^{2}+z^{2}\right)^{\frac{9}{2}}+\right. \\
4.375\left(z-\sqrt{x^{2}+y^{2}+z^{2}}\right)^{4}\left(x^{2}+y^{2}+z^{2}\right)^{3}+17.5\left(z-\sqrt{x^{2}+y^{2}+z^{2}}\right)^{3}\left(x^{2}+y^{2}+z^{2}\right)^{\frac{7}{2}}+ \\
\left.22.5\left(z-\sqrt{x^{2}+y^{2}+z^{2}}\right)^{2}\left(x^{2}+y^{2}+z^{2}\right)^{4}-9.0\left(x^{2}+y^{2}+z^{2}\right)^{5}\right) \sqrt{\frac{1}{a^{3}}} e^{-\frac{\sqrt{x^{2}+y^{2}+z^{2}}}{7 a}} \div\left(\sqrt{\pi} a^{6}\left(x^{2}+\right.\right. \\
\left.\left.y^{2}+z^{2}\right)^{3}\right)\end{array}$ & 42 \\
\hline $\begin{array}{l}\Psi_{(7,5,0)} \quad=1.1726 \sqrt{2}\left(4.26095 \cdot 10^{-8} a-1.014512 \cdot 10^{-9} \sqrt{x^{2}+y^{2}+z^{2}}\right)\left(15.0 z\left(x^{2}+y^{2}+\right.\right. \\
\left.z^{2}\right)^{7}+7.875\left(z-\sqrt{x^{2}+y^{2}+z^{2}}\right)^{5}\left(x^{2}+y^{2}+z^{2}\right)^{5}+39.375\left(z-\sqrt{x^{2}+y^{2}+z^{2}}\right)^{4}\left(x^{2}+y^{2}+\right. \\
\left.z^{2}\right)^{\frac{11}{2}}+70.0\left(z-\sqrt{x^{2}+y^{2}+z^{2}}\right)^{3}\left(x^{2}+y^{2}+z^{2}\right)^{6}+52.5\left(z-\sqrt{x^{2}+y^{2}+z^{2}}\right)^{2}\left(x^{2}+y^{2}+z^{2}\right)^{\frac{13}{2}}- \\
\left.14.0\left(x^{2}+y^{2}+z^{2}\right)^{\frac{15}{2}}\right) \sqrt{\frac{1}{a^{3}}} e^{-\frac{\sqrt{x^{2}+y^{2}+z^{2}}}{7 a}} \div\left(\sqrt{\pi} a^{6}\left(x^{2}+y^{2}+z^{2}\right)^{5}\right)\end{array}$ & 43 \\
\hline $\begin{array}{l}\Psi_{(7,6,0)}=\sqrt{2}\left(7.5323669 \cdot 10^{-9} z\left(x^{2}+y^{2}+z^{2}\right)^{10}+5.1785023 \cdot 10^{-9}\left(z-\sqrt{x^{2}+y^{2}+z^{2}}\right)^{6}\left(x^{2}+\right.\right. \\
\left.y^{2}+z^{2}\right)^{\frac{15}{2}}+3.1071014 \cdot 10^{-8}\left(z-\sqrt{x^{2}+y^{2}+z^{2}}\right)^{5}\left(x^{2}+y^{2}+z^{2}\right)^{8}+7.061594 \cdot 10^{-8}(z- \\
\left.\sqrt{x^{2}+y^{2}+z^{2}}\right)^{4}\left(x^{2}+y^{2}+z^{2}\right)^{\frac{17}{2}}+7.532367 \cdot 10^{-8}\left(z-\sqrt{x^{2}+y^{2}+z^{2}}\right)^{3}\left(x^{2}+y^{2}+z^{2}\right)^{9}+ \\
3.7662 \cdot 10^{-8}\left(z-\sqrt{x^{2}+y^{2}+z^{2}}\right)^{2}\left(x^{2}+y^{2}+z^{2}\right)^{\frac{19}{2}}-7.173683 \cdot \\
\left.\left(x^{2}+y^{2}+z^{2}\right)^{\frac{21}{2}}\right) \sqrt{\frac{1}{a^{3}}} e^{-\frac{\sqrt{x^{2}+y^{2}+z^{2}}}{7 a}} \div\left(\sqrt{\pi} a^{6}\left(x^{2}+y^{2}+z^{2}\right)^{\frac{15}{2}}\right)\end{array}$ & 44 \\
\hline $\begin{array}{l}\Psi_{(8,0,0)} \quad=\sqrt{2}\left(0.03125 a^{7}-0.02734375 a^{6} \sqrt{x^{2}+y^{2}+z^{2}}+0.006836 a^{5}\left(x^{2}+y^{2}+z^{2}\right)-\right. \\
0.0007120768 a^{4}\left(x^{2}+y^{2}+z^{2}\right)^{\frac{3}{2}}+3.56038411458333 \cdot 10^{-5} a^{3}\left(x^{2}+y^{2}+z^{2}\right)^{2}- \\
8.90096028645833 \cdot 10^{-7} a^{2}\left(x^{2}+y^{2}+z^{2}\right)^{\frac{5}{2}}+1.05963812934028 \cdot 10^{-8} a\left(x^{2}+y^{2}+z^{2}\right)^{3}- \\
\left.4.73052736312624 \cdot 10^{-11}\left(x^{2}+y^{2}+z^{2}\right)^{\frac{7}{2}}\right) \sqrt{\frac{1}{a^{3}}} e^{-\frac{\sqrt{x^{2}+y^{2}+z^{2}}}{8 a}} \div\left(\sqrt{\pi} a^{7}\right)\end{array}$ & 45 \\
\hline 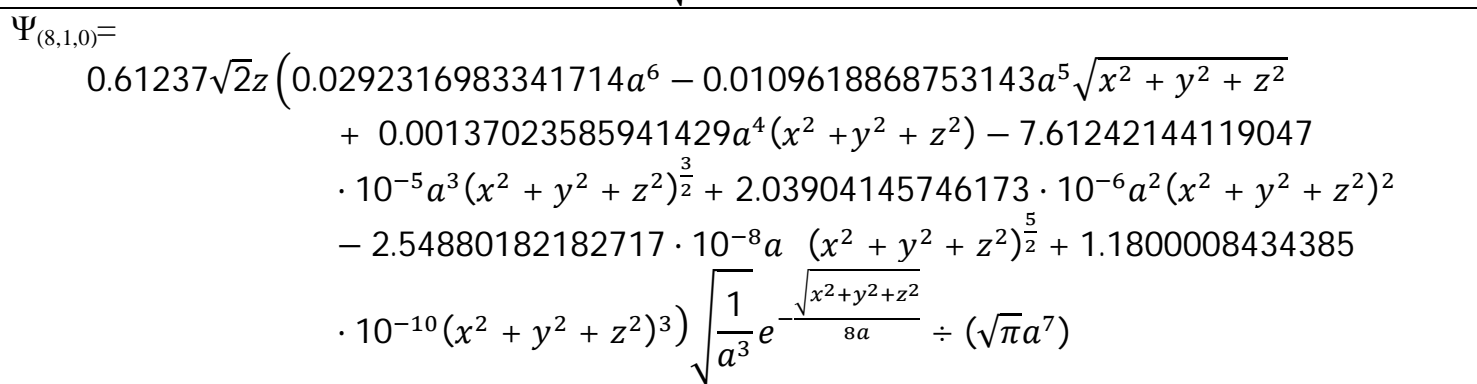 & 46 \\
\hline $\begin{array}{l}\Psi_{(8,2,0)}=0.790569415042095 \sqrt{2}\left(3.0 z\left(x^{2}+y^{2}+z^{2}\right)+1.5\left(z-\sqrt{x^{2}+y^{2}+z^{2}}\right)^{2} \sqrt{x^{2}+y^{2}+z^{2}}-\right. \\
\left.2.0\left(x^{2}+y^{2}+z^{2}\right)^{\frac{3}{2}}\right)\left(0.00283034702074012 a^{5}-0.000589655629320859 a^{4} \sqrt{x^{2}+y^{2}+z^{2}}+\right. \\
4.21182592372042 \times 10^{-5} a^{3}\left(x^{2}+y^{2}+z^{2}\right)-1.31619560116263 \cdot \times 10^{-6} a^{2}\left(x^{2}+y^{2}+\right. \\
\left.z^{2}\right)^{\frac{3}{2}}+1.82804944605921 \cdot 10^{-8} a\left(x^{2}+y^{2}+z^{2}\right)^{2}-9.14024723029606 \cdot 10^{-11}\left(x^{2}+y^{2}+\right. \\
\left.\left.z^{2}\right)^{\frac{5}{2}}\right) \sqrt{\frac{1}{a^{3}}} e^{-\frac{\sqrt{x^{2}+y^{2}+z^{2}}}{8 a}} \div\left(\sqrt{\pi} a^{7} \sqrt{x^{2}+y^{2}+z^{2}}\right)\end{array}$ & 47 \\
\hline $\begin{array}{l}\Psi_{(8,3,0)}=0.935414346693485 \sqrt{2}\left(6.0 z\left(x^{2}+y^{2}+z^{2}\right)^{\frac{5}{2}}+2.5\left(z-\sqrt{x^{2}+y^{2}+z^{2}}\right)^{3}\left(x^{2}+y^{2}+z^{2}\right)^{\frac{3}{2}}+\right. \\
\left.7.5\left(z-\sqrt{x^{2}+y^{2}+z^{2}}\right)^{2}\left(x^{2}+y^{2}+z^{2}\right)^{2}-5.0\left(x^{2}+y^{2}+z^{2}\right)^{3}\right)\left(0.000124942948173623 a^{4}-\right. \\
1.5617868 \times 10^{-5} a^{3} \sqrt{x^{2}+y^{2}+z^{2}}+6.50744521737619 \cdot 10^{-7} a^{2}\left(x^{2}+y^{2}+z^{2}\right)- \\
1.08457420289603 \cdot 10^{-8} a\left(x^{2}+y^{2}+z^{2}\right)^{\frac{3}{2}}+6.16235342554563 \cdot 10^{-11}\left(x^{2}+y^{2}+\right.\end{array}$ & 48 \\
\hline
\end{tabular}




\begin{tabular}{|c|c|}
\hline$\left.\left.z^{2}\right)^{2}\right) \sqrt{\frac{1}{a^{3}}} e^{-\frac{\sqrt{x^{2}+y^{2}+z^{2}}}{8 a}} \div\left(\sqrt{\pi} a^{7}\left(x^{2}+y^{2}+z^{2}\right)^{\frac{3}{2}}\right)$ & \\
\hline $\begin{array}{l}\Psi_{(8,4,0)}= \\
1.06066017177982 \sqrt{2}\left(3.00566019839111 \cdot 10^{-6} a^{3}-2.25424514879333\right. \\
10^{-7} a^{2} \sqrt{x^{2}+y^{2}+z^{2}}+5.12328442907575 \cdot 10^{-9} a\left(x^{2}+y^{2}+z^{2}\right)-3.55783640908038 \\
\left.10^{-11}\left(x^{2}+y^{2}+z^{2}\right)^{\frac{3}{2}}\right)\left(10.0 z\left(x^{2}+y^{2}+z^{2}\right)^{\frac{9}{2}}+4.375\left(z-\sqrt{x^{2}+y^{2}+z^{2}}\right)^{4}\left(x^{2}+y^{2}+z^{2}\right)^{3}+\right. \\
17.5\left(z-\sqrt{x^{2}+y^{2}+z^{2}}\right)^{3}\left(x^{2}+y^{2}+z^{2}\right)^{\frac{7}{2}}+22.5\left(z-\sqrt{x^{2}+y^{2}+z^{2}}\right)^{2}\left(x^{2}+y^{2}+z^{2}\right)^{4}- \\
\left.9.0\left(x^{2}+y^{2}+z^{2}\right)^{5}\right) \sqrt{\frac{1}{a^{3}}} e^{-\frac{\sqrt{x^{2}+y^{2}+z^{2}}}{8 a}} \div\left(\sqrt{\pi} a^{7}\left(x^{2}+y^{2}+z^{2}\right)^{3}\right)\end{array}$ & 49 \\
\hline $\begin{array}{l}\Psi_{(8,5,0)}= \\
1.17260393995586 \sqrt{2}\left(4.2659868006405 \cdot 10^{-8} a^{2}-1.77749450026687\right. \\
10^{-9} a \sqrt{x^{2}+y^{2}+z^{2}}+1.70912932717969 \cdot 10^{-11} x^{2}+1.70912932717969 \cdot 10^{-11} y^{2}+ \\
\left.1.70912932717969 \cdot 10^{-11} z^{2}\right)\left(15.0 z\left(x^{2}+y^{2}+z^{2}\right)^{7}+7.875\left(z-\sqrt{x^{2}+y^{2}+z^{2}}\right)^{5}\left(x^{2}+y^{2}+\right.\right. \\
\left.z^{2}\right)^{5}+39.375\left(z-\sqrt{x^{2}+y^{2}+z^{2}}\right)^{4}\left(x^{2}+y^{2}+z^{2}\right)^{\frac{11}{2}}+70.0\left(z-\sqrt{x^{2}+y^{2}+z^{2}}\right)^{3}\left(x^{2}+y^{2}+\right. \\
\left.\left.z^{2}\right)^{6}+52.5\left(z-\sqrt{x^{2}+y^{2}+z^{2}}\right)^{2}\left(x^{2}+y^{2}+z^{2}\right)^{\frac{13}{2}}-14.014 .0\left(x^{2}+y^{2}+z^{2}\right)^{\frac{15}{2}}\right) \sqrt{\frac{1}{a^{3}}} e^{-\frac{\sqrt{x^{2}+y^{2}+z^{2}}}{8 a}} \div \\
\left(\sqrt{\pi} a^{7}\left(x^{2}+y^{2}+z^{2}\right)^{5}\right)\end{array}$ & 50 \\
\hline $\begin{array}{l}\Psi_{(8,6,0)}= \\
1.2747548783982 \sqrt{2}\left(3.61754492653168 \cdot 10^{-10} a-6.45990165452086\right. \\
\left.10^{-12} \sqrt{x^{2}+y^{2}+z^{2}}\right)\left(21.0 z\left(x^{2}+y^{2}+z^{2}\right)^{10}+14.4375\left(z-\sqrt{x^{2}+y^{2}+z^{2}}\right)^{6}\left(x^{2}+y^{2}+z^{2}\right)^{\frac{15}{2}}+\right. \\
86.625\left(z-\sqrt{x^{2}+y^{2}+z^{2}}\right)^{5}\left(x^{2}+y^{2}+z^{2}\right)^{8}+196.875\left(z-\sqrt{x^{2}+y^{2}+z^{2}}\right)^{4}\left(x^{2}+y^{2}+z^{2}\right)^{\frac{17}{2}}+ \\
210.0\left(z-\sqrt{x^{2}+y^{2}+z^{2}}\right)^{3}\left(x^{2}+y^{2}+z^{2}\right)^{9}+105.0\left(z-\sqrt{x^{2}+y^{2}+z^{2}}\right)^{2}\left(x^{2}+y^{2}+z^{2}\right)^{\frac{19}{2}}- \\
\left.20.0\left(x^{2}+y^{2}+z^{2}\right)^{\frac{21}{2}}\right) \sqrt{\frac{1}{a^{3}}} e^{-\frac{\sqrt{x^{2}+y^{2}+z^{2}}}{8 a}} \div\left(\sqrt{\pi} a^{7}\left(x^{2}+y^{2}+z^{2}\right)^{\frac{15}{2}}\right)\end{array}$ & 51 \\
\hline $\begin{array}{l}\Psi_{(8,7,0)} \quad=\sqrt{2}\left(6.39497637199385 \cdot 10^{-11} z\left(x^{2}+y^{2}+z^{2}\right)^{\frac{27}{2}}-2.79907421453732 \cdot 10^{-12}(z-\right. \\
\left.\sqrt{x^{2}+y^{2}+z^{2}}\right)^{7}\left(x^{2}+y^{2}+z^{2}\right)^{\frac{21}{2}}+1.33001057808236 \cdot 10^{-10}\left(z-\sqrt{x^{2}+y^{2}+z^{2}}\right)^{6}\left(x^{2}+y^{2}+\right. \\
\left.z^{2}\right)^{11}+1.18706748905136 \cdot 10^{-9}\left(z-\sqrt{x^{2}+y^{2}+z^{2}}\right)^{5}\left(x^{2}+y^{2}+z^{2}\right)^{\frac{23}{2}}+1.6487 \times 10^{-9}(z- \\
\left.\sqrt{x^{2}+y^{2}+z^{2}}\right)^{4}\left(x^{2}+y^{2}+z^{2}\right)^{12}+1.19905806974885 \cdot 10^{-9}\left(z-\sqrt{x^{2}+y^{2}+z^{2}}\right)^{3}\left(x^{2}+y^{2}+\right. \\
\left.z^{2}\right)^{\frac{25}{2}}+4.31660905109585 \cdot 10^{-10}\left(z-\sqrt{x^{2}+y^{2}+z^{2}}\right)^{2}\left(x^{2}+y^{2}+z^{2}\right)^{13}-6.16658435870836 . \\
\left.10^{-11}\left(x^{2}+y^{2}+z^{2}\right)^{14}\right) \sqrt{\frac{1}{a^{3}}} e^{-\frac{\sqrt{x^{2}+y^{2}+z^{2}}}{8 a}} \div\left(\sqrt{\pi} a^{7}\left(x^{2}+y^{2}+z^{2}\right)^{\frac{21}{2}}\right)\end{array}$ & 52 \\
\hline $\begin{array}{l}\Psi_{(9,0,0)} \\
\sqrt{2}\left(0.0261891400439462 a^{8}-0.0232792355946188 a^{7} \sqrt{x^{2}+y^{2}+z^{2}}+\right. \\
\quad 0.00603535737638266 a^{6}\left(x^{2}+y^{2}+z^{2}\right)- \\
0.000670595264042518 a^{5}\left(x^{2}+y^{2}+z^{2}\right)^{\frac{3}{2}}+3.72552924468066 \cdot 10^{-5} a^{4}\left(x^{2}+y^{2}+z^{2}\right)^{2}- \\
1.10386051694242 \cdot 10^{-6} a^{3}\left(x^{2}+y^{2}+z^{2}\right)^{\frac{5}{2}}+1.75215955070225 \cdot 10^{-8} a^{2}\left(x^{2}+y^{2}+z^{2}\right)^{3}- \\
1.39060281801766 \quad \cdot 10^{-10} a\left(x^{2}+y^{2}+z^{2}\right)^{\frac{7}{2}}+4.29198400622734 \cdot 10^{-13}\left(x^{2}+\right. \\
\left.\left.y^{2}+z^{2}\right)^{4}\right) \sqrt{\frac{1}{a^{3}}} e^{-\frac{\sqrt{x^{2}+y^{2}+z^{2}}}{9 a}} \div\left(\sqrt{\pi} a^{8}\right)\end{array}$ & 53 \\
\hline $\begin{array}{l}\Psi_{(9,1,0)}= \\
0.612372435695794 \sqrt{2} z\left(0.0245384688888866 a^{7}-0.00954273790123367 a^{6} \sqrt{x^{2}+y^{2}+z^{2}}+\right. \\
0.00127236505349782 a^{5}\left(x^{2}+y^{2}+z^{2}\right)-7.85410526850508 \cdot 10^{-5} a^{4}\left(x^{2}+y^{2}+z^{2}\right)^{\frac{3}{2}}+ \\
2.49336675190637 \cdot 10^{-6} a^{3}\left(x^{2}+y^{2}+z^{2}\right)^{2}-4.15561125317729 \cdot 10^{-8} a^{2}\left(x^{2}+y^{2}+z^{2}\right)^{\frac{5}{2}}+ \\
3.42025617545456 \cdot 10^{-10} a\left(x^{2}+y^{2}+z^{2}\right)^{3}-1.08579561125542 \\
\end{array}$ & 54 \\
\hline
\end{tabular}




\begin{tabular}{|l|l|}
\hline$\left.\left.z^{2}\right)^{\frac{7}{2}}\right) \sqrt{\frac{1}{a^{3}}} e^{-\frac{\sqrt{x^{2}+y^{2}+z^{2}}}{9 a}} \div\left(\sqrt{\pi} a^{8}\right)$ & \\
\hline$\Psi_{(9,2,0)}=0.790569415042095 \sqrt{2}\left(3.0 z\left(x^{2}+y^{2}+z^{2}\right)+1.5\left(z-\sqrt{x^{2}+y^{2}+z^{2}}\right)^{2} \sqrt{x^{2}+y^{2}+z^{2}}-\right.$ & 55 \\
$\left.2.0\left(x^{2}+y^{2}+z^{2}\right)^{\frac{3}{2}}\right)\left(0.00239249100690121 a^{6}-0.00053166466820027 a^{5} \sqrt{x^{2}+y^{2}+z^{2}}+\right.$ & \\
$4.2195608587323 \cdot 10^{-5} a^{4}\left(x^{2}+y^{2}+z^{2}\right)-1.562800318049 \cdot 10^{-6} a^{3}\left(x^{2}+y^{2}+z^{2}\right)^{\frac{3}{2}}+$ & \\
$2.8940746630537 \cdot 10^{-8} a^{2}\left(x^{2}+y^{2}+z^{2}\right)^{2}-2.57251081160329 \cdot 10^{-10} a\left(x^{2}+y^{2}+z^{2}\right)^{\frac{5}{2}}+$ & \\
$\left.8.66165256432085 \cdot 10^{-13}\left(x^{2}+y^{2}+z^{2}\right)^{3}\right) \sqrt{\frac{1}{a^{3}}} e^{-\frac{\sqrt{x^{2}+y^{2}+z^{2}}}{9 a}} \div\left(\sqrt{\pi} a^{8} \sqrt{x^{2}+y^{2}+z^{2}}\right)$ & \\
\hline$\Psi_{(9,3,0)}=0.935414346693485 \sqrt{2}\left(6.0 z\left(x^{2}+y^{2}+z^{2}\right)^{\frac{5}{2}+2.5\left(z-\sqrt{x^{2}+y^{2}+z^{2}}\right)^{3}\left(x^{2}+y^{2}+z^{2}\right)^{\frac{3}{2}}+}\right.$ & 56 \\
$\left.7.5\left(z-\sqrt{x^{2}+y^{2}+z^{2}}\right)^{2}\left(x^{2}+y^{2}+z^{2}\right)^{2}-5.0\left(x^{2}+y^{2}+z^{2}\right)^{3}\right)\left(0.000107412483486202 a^{5}-\right.$ & \\
$1.49184004841947 \cdot 10^{-5} a^{4} \sqrt{x^{2}+y^{2}+z^{2}}+7.36711135021961 \cdot 10^{-7} a^{3}\left(x^{2}+y^{2}+z^{2}\right)-$ & \\
$1.63713585560436 \cdot 10^{-8} a^{2}\left(x^{2}+y^{2}+z^{2}\right)^{\frac{3}{2}}+1.65367258141854 \cdot 10^{-10} a\left(x^{2}+y^{2}+z^{2}\right)^{2}-$ & \\
$\left.6.12471326451312 \cdot 10^{-13}\left(x^{2}+y^{2}+z^{2}\right)^{\frac{5}{2}}\right) \sqrt{\frac{1}{a^{3}}} e^{-\frac{\sqrt{x^{2}+y^{2}+z^{2}}}{9 a}} \div\left(\sqrt{\pi} a^{8}\left(x^{2}+y^{2}+z^{2}\right)^{\frac{3}{2}}\right.$ & \\
\hline
\end{tabular}

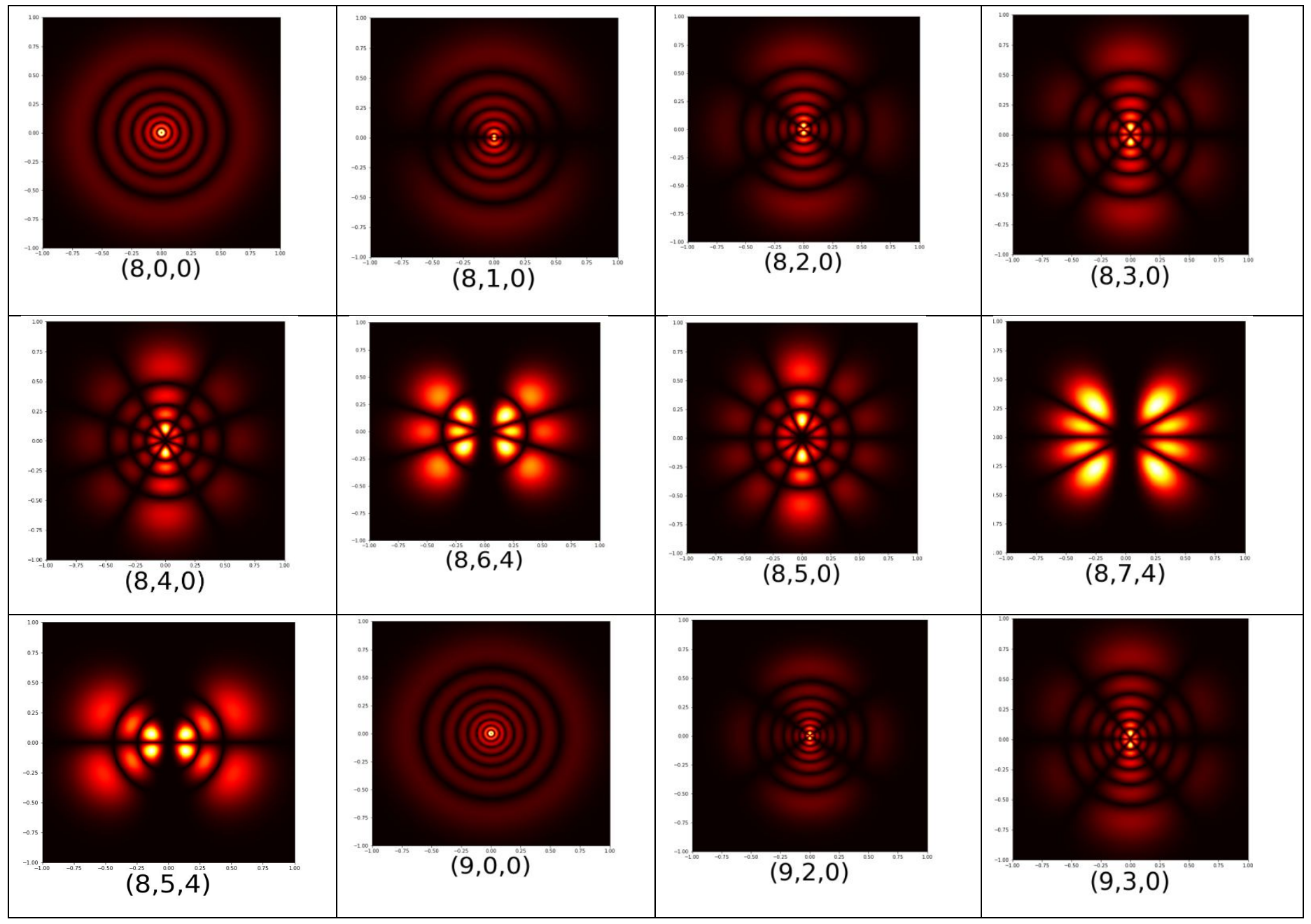




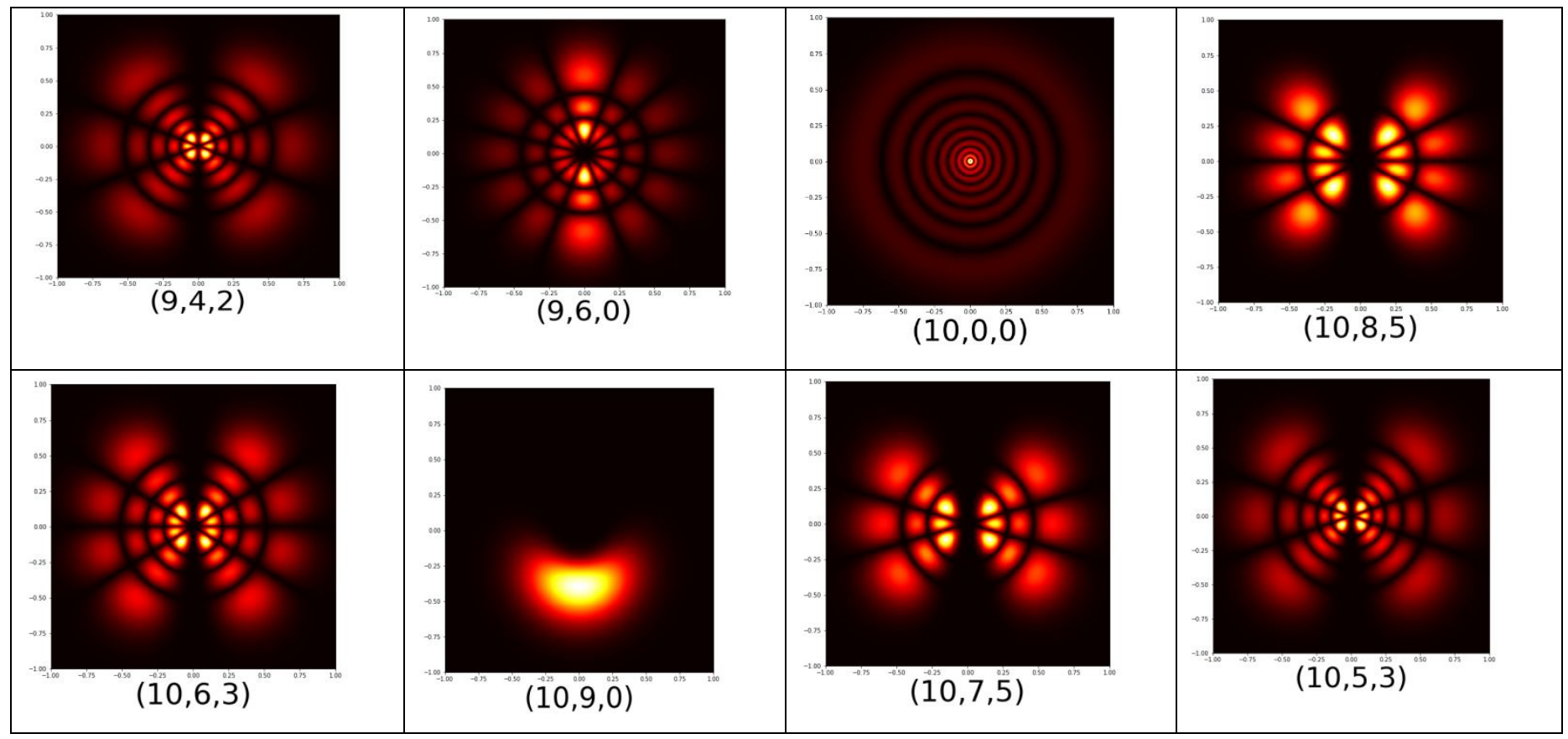

Figure 1: Few wave-functions plotting in Cartesian co-ordinate

\section{CONCLUSION}

Pictorial and analytical form of Hydrogen wave function in Cartesian coordinate is a base to calculate wave function, probability density, total energy etc of many theoretical methods to solve many electrons atoms. reported python code can be used to calculate many properties like size, energies with growing atomic size, orbit number of orbital etc. we can also use wave functions in Cartesian coordinates to visualized and understand behavior of orbitals. In this work 40 wave function in analytical and 20 probability distribution, functions in pictorial form in Cartesian coordinates are reported.

\section{REFERENCES}

1. Budde, M., Niedderer, H., Scott, P., \& Leach, J.. 'Electronium': a quantum atomic teaching model. Physics Education, 37(3), 197,2002 .

2.Budde, M., Niedderer, H., Scott, P., \& Leach, J. . The quantum atomic model 'Electronium': a successful teaching tool. Physics Education, 37(3), 204,2002.

3.Müller, R., \& Wiesner, H. . Teaching quantum mechanics on an introductory level. American Journal of physics, 70(3), 200$209,2002$.

4.Niedderer, H., \& Deylitz, S. Evaluation of a new approach in quantum atomic physics in high school. In Annual Meeting National Association For Research In Science Teaching, Boston.
Collection of papers presented. Disponible en internet: www. phys. ksu. edu/perg/papers/narst. March 1999.

5.Fowles, G. R. Solution of the Schrödinger equation for the hydrogen atom in rectangular coordinates. American Journal of Physics, 30(4), 308-309, 1962.

6.Wang, J. B., Abbott, P. C., \& Williams, J. F.. Visualizing atomic orbitals. Computers in Physics, 10(1), 69-82,1996.

7. Chung, W. C.Three-dimensional atomic orbital plots in the classroom using Winplot 2013

8. Chung, W. C. Three-dimensional atomic orbital plots in the classroom using Winplot.2013.

9. Griffiths, D. J. Introduction to quantum mechanics. Prentice Hall, second edi.2010.

10. Liboff, R. L. Introductory quantum mechanics. Pearson Education India.2003.

11. Zettili, N. (2003). Quantum mechanics: concepts and applications.

12. Condon, E. U., \& Odabasi, H. Atomic structure. CUP Archive1980.

13. Fischer, C. F. Hartree--Fock method for atoms. A numerical approach 1977.

14. Arfken, G. B., \& Weber, H. J. (). Mathematical methods for physicists , 1999 .

15. Ahmed Ali Rajput, Fida Hussain Khoso, Muhammad Daniyal, Misha Shafi, Muhammad MustaqeemZahid, Hasan Nafees and Zaheer Uddin,A python program to model and analyze wind speed data. International Journal, 9(6)2021. 
Kousar Shaheen et al., International Journal of Advanced Trends in Computer Science and Engineering, 10(3), May - June 2021,2513 - 2523

\section{Appendix A}

\section{Python code}

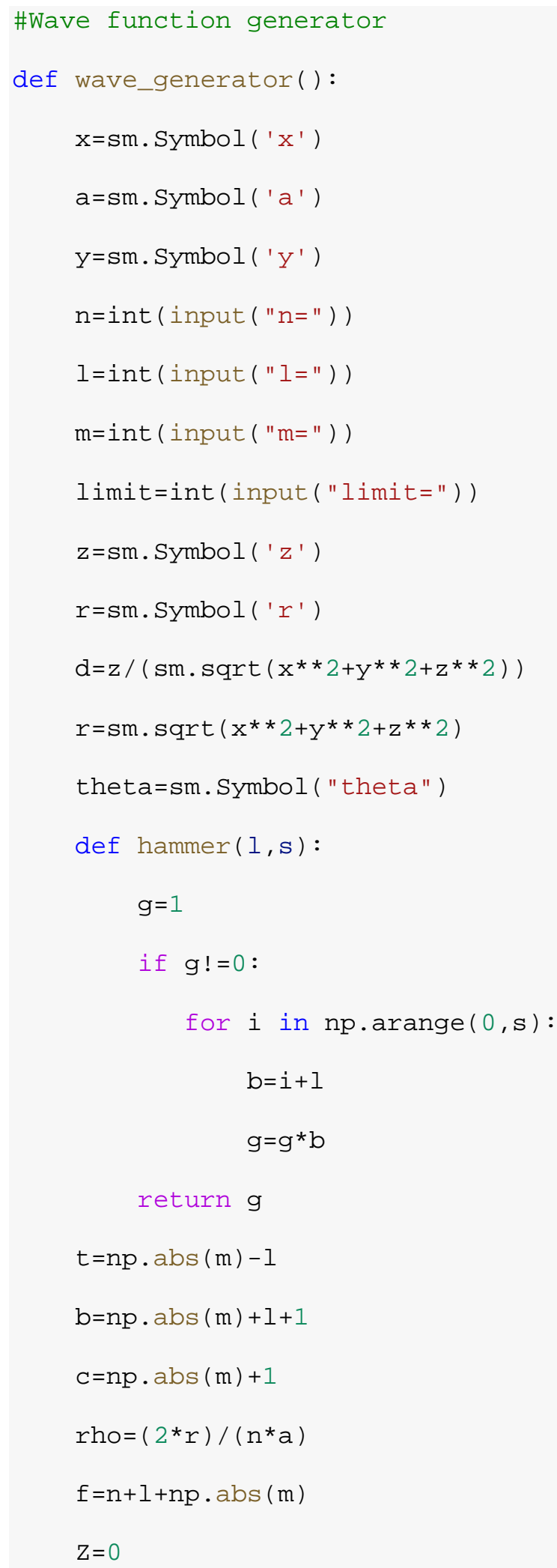

$\mathrm{R}=1$

$\mathrm{p}=\mathrm{sm} \cdot \mathrm{pi}$

$Q=\operatorname{sm} . \operatorname{sqrt}(1 /(2 \star p))$

$\mathrm{U}=\mathrm{sm} \cdot \operatorname{sqrt}((((2 / \mathrm{n}) \star \star 3))) * \mathrm{sm} \cdot \operatorname{sqrt}((((\mathrm{mt} \cdot \mathrm{fa}$ ctorial (n-l1) ) $/((2 * n) *($ mt . factorial $(n+1) * \star 3)))))$ * Fract ion $(2 / \mathrm{n}) * \star 1) * \mathrm{sm} \cdot \exp (-$

$$
\mathrm{T}=\mathrm{simplify}\left(\mathrm{F}^{\star} \mathrm{O}\right)
$$


Kousar Shaheen et al., International Journal of Advanced Trends in Computer Science and Engineering, 10(3), May - June 2021,2513 - 2523

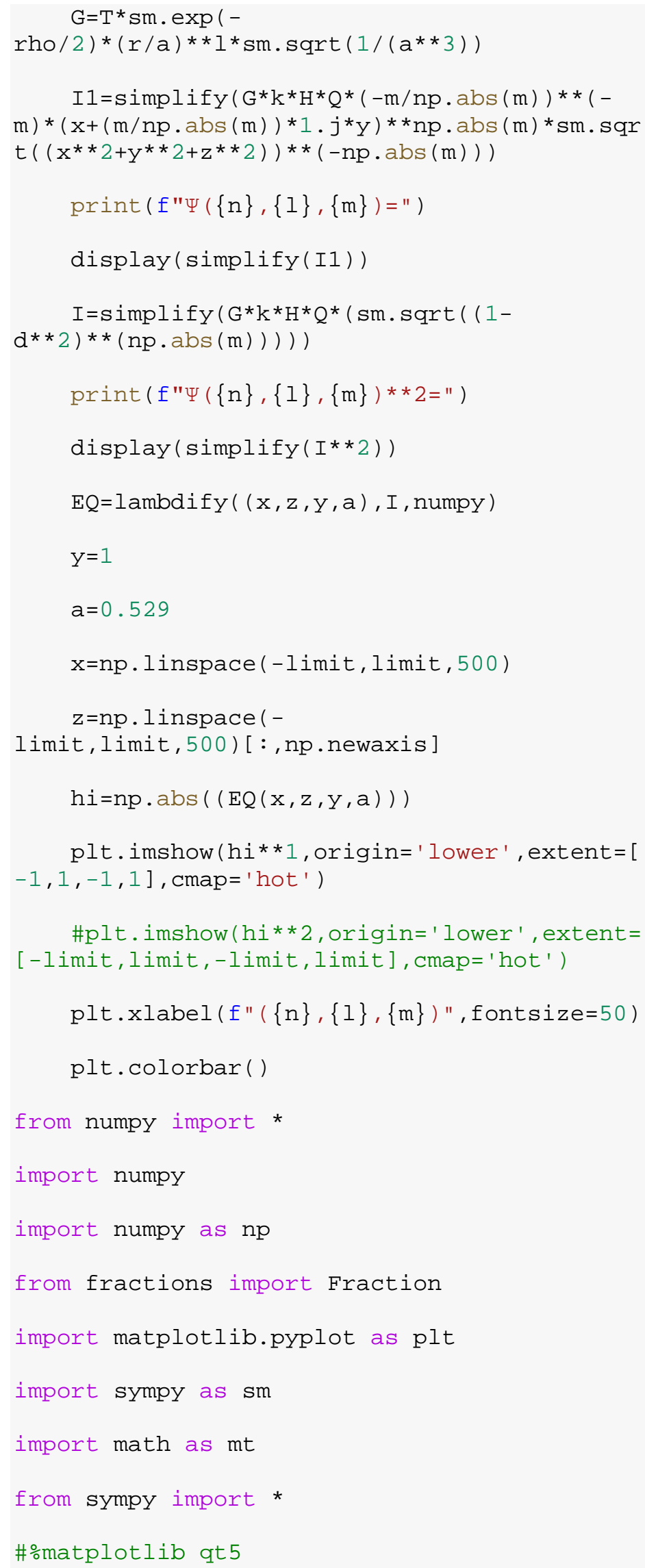

sm.init_printing (use_latex=True)

print (wave_generator()) 